\title{
Role of therapeutic plasma exchange in acute liver failure due to yellow phosphorus poisoning
}

\author{
Joy Varghese ${ }^{1}$ - Vivek Joshi ${ }^{1}$. Madhan Kumar Bollipalli ${ }^{2}$. Selvakumar Malleeswaran ${ }^{3} \cdot$ Rajinikanth Patcha $^{4}$. \\ Harikumar Nair ${ }^{1}$ - Vivek Vij $^{4}$ • Deepti Sachan ${ }^{5}$. Pushkala Subramanian ${ }^{6}$ - Mayank Jain ${ }^{1}$. Jayanthi Venkataraman ${ }^{7}$
}

Received: 12 March 2020 / Accepted: 14 September 2020 / Published online: 6 January 2021

(C) Indian Society of Gastroenterology 2021

\begin{abstract}
Background Therapeutic plasma exchange (TPE) has been utilized in various liver disorders. There is limited data on the efficacy of TPE in patients with acute liver failure (ALF).

Methods Study group consisted of patients who underwent TPE for ALF due to yellow phosphorous poisoning (YPP) between 2015 and 2019. Demographic data and biochemical parameters were recorded before and after TPE. Overall survival and transplant-free survival (based on King's College Hospital Criteria $[\mathrm{KCHC}]$ ) were analyzed.

Results Forty-three patients underwent TPE for ALF due to YPP. Most of them were young males. Overall survival was 34 (79.06\%). In our study population, 20 patients fulfilled KCHC (Group A) and 23 did not fulfill KCHC (Group B). Both the groups showed significant improvement in alanine aminotransferase, aspartate aminotransferase, and international normalized ratio (INR) after TPE $(p<0.05)$. In Group B, there was significant improvement in ammonia after TPE $(p<0.05)$ and all 23 patients $(100 \%)$ survived after TPE. In Group A, 4 underwent liver transplantation (LT), 7 survived without LT, and the remaining 9 died without LT. Mean survival after completing TPE was 41.2 \pm 44.5 days in Group A and 90 days in Group B. This difference was statistically significant $(p=0.001)$. There was statistically significant difference in post-TPE values of INR $(p=0.012)$ and ammonia $(p=0.011)$ between non-survivors and survivors. Adverse events such as hypotension $(11.62 \%)$ and minor allergic reaction $(4.65 \%)$ were managed conservatively.

Conclusion TPE is an effective procedure in ALF due to YPP, not fulfilling KCHC for LT. In KCHC fulfilled group, though it shows LT-free survival benefit, there is requirement of prospective, large volume, multi-center study to assess its efficacy.
\end{abstract}

Keywords Fulminant liver failure $\cdot$ Hepatic failure $\cdot$ Liver transplantation $\cdot \mathrm{N}$-acetyl cysteine $\cdot$ Plasmapheresis $\cdot$ Ratol poisoning

Vivek Joshi

vivekjoshi91@gmail.com

1 Department of Hepatology and Transplant Hepatology, Institute of Liver Diseases and Transplantation, Gleneagles Global Health City, Chennai 600 100, India

2 Department of Transfusion Medicine, Gleneagles Global Health City, Chennai 600 100, India

3 Department of Liver Anesthesia and ICU, Gleneagles Global Health City, Chennai 600 100, India

4 Department of HPB and Transplantation, Gleneagles Global Health City, Chennai 600 100, India

5 Department of Transfusion Medicine, Dr. Rela Institute and Medical Centre, Chennai 600 044, India

6 Department of Immunology, The Tamil Nadu Dr. MGR University, Chennai 600 032, India

7 Department of Hepatology, Sri Ramachandra Institute for Higher Education and Research, Chennai 600 116, India

\section{Introduction}

Phosphorus has several allotropes, which exhibit strikingly diverse properties. White phosphorus and red phosphorus are the two most common allotropes of phosphorus. White phosphorus is a translucent waxy solid which quickly gets converted to yellow when exposed to light. For this reason, it is also known as yellow phosphorus (YP) [1]. Yellow phosphorus is used in production of rodenticides, fireworks, and fertilizers and it is a general protoplasmic toxin [2]. Rodenticides are available as pastes or powders, which contain around $2 \%$ to $5 \%$ yellow phosphorus. This is a highly toxic compound which can cause hepatocellular necrosis and fulminant hepatic failure. The estimated lethal dose of yellow phosphorus is $1 \mathrm{mg} / \mathrm{kg}$ that results in death due to acute liver failure (ALF) and cardiovascular collapse [3].

Therapeutic plasma exchange (TPE) is defined as the removal of patient's plasma and replacing it with plasma from a 


\section{Bullet points of the study highlights}

\section{What is already known?}

- Therapeutic plasma exchange (TPE) can be used in various liver diseases including acute liver failure (ALF).

- In patients with ALF due to yellow phosphorus poisoning (YPP), liver transplantation is the only definitive cure if King's College Hospital Criteria $(\mathrm{KCHC})$ is getting fulfilled.

\section{What is new in this study?}

- We have assessed role of TPE in patients with ALF due to YPP.

- We found that $43.8 \%$ patients with ALF due to YPP who met KCHC got delisted from the liver transplantation list with TPE.

\section{What are the future clinical and research implications of the study findings?}

- TPE can be curative in a subgroup of patients with ALF due to YPP who meet KCHC.

- Prospective, large volume, multi-center study should further assess its efficacy in ALF due to YPP and other liver disorders.

donor along with colloid by using an extracorporeal device. It is an effective method for the removal of accumulated toxins from plasma in liver failure patients $[4,5]$. Though TPE reduces blood ammonia levels, it has an added advantage of providing deficient clotting factors and albumin. There is limited data on the efficacy of TPE in patients with ALF. We report outcome of TPE in 43 patients who presented with ALF due to yellow phosphorous poisoning (YPP).

\section{Methods (Fig. 1)}

All patients belonging to either gender, presenting to Gleneagles Global Health City, Chennai, with ALF due to YPP, between 2015 and 2019, were included in this retrospective study. Patients who had taken YP along with alcohol, paracetamol, or other medications were excluded. ALF was defined as "a severe acute liver injury with encephalopathy and impaired synthetic function (international normalized ratio [INR] of $\geq 1.5$ ) in a patient without preexisting cirrhosis or liver disease and with an illness of $<26$ weeks' duration" [6]. Baseline information obtained included demographic, clinical, and biochemical parameters.

Protocol-based management was introduced for YPP presenting as ALF. Patients were admitted in liver intensive care unit (LICU) from time of registration. Vitals (pulse, blood pressure, temperature, respiratory rate), level of consciousness, weight, serum electrolytes (sodium, potassium, magnesium, calcium), blood glucose, and hourly urine output were monitored at regular intervals.

Standard care was provided to all the patients; these included prophylactic intravenous antibiotics, parenteral vitamin K, proton pump inhibitor, correction of dyselectrolytemia, and intravenous fluids. Serum sodium was maintained between 145 and $155 \mathrm{mmol} / \mathrm{L}$ with hypertonic saline [6]. Vasopressors were introduced, need based, in order to maintain a mean arterial pressure of $60 \mathrm{mmHg}$ [7]. Grade III/IV encephalopathy necessitated mechanical ventilator support. Use of $\mathrm{N}$-acetyl cysteine has been found to be useful in patients with ALF due to YPP $[8,9]$. We used $\mathrm{N}$-acetyl cysteine in a dose of $14 \mathrm{~g} /$ day for 5 consecutive days according to our unit protocol.

Study group underwent TPE as earlier studies showed benefit of TPE in patients with ALF [5]. Spectra Optia ${ }^{\circledR}$ (Terumo BCT, Colorado, USA) machine was used with centrifugation set at 3000 revolution per minute (RPM). TPE was offered once a day for 5 consecutive days under constant monitoring of vital parameters. One plasma volume was exchanged for each cycle of TPE with replacement fluid, which was constituted with fresh frozen plasma and 5\% human albumin in equal fractions. Replacement volume during each cycle of TPE was calculated based on patient's height, weight and 
Fig. 1 Flow diagram on study cohort of yellow phosphorus poisoning presenting as acute liver failure. $Y P P$ yellow phosphorus poisoning, $A L F$ acute liver failure, $\mathrm{KCHC}$ King's College hospital criteria, $L I C U$ liver intensive care unit, $I V$ intravenous, $P P I$ proton pump inhibitor, $N A C \mathrm{~N}$-acetyl cysteine, $M A P$ mean arterial pressure, $H E$ hepatic encephalopathy, $T P E$ therapeutic plasma exchange, $L T$ liver transplantation

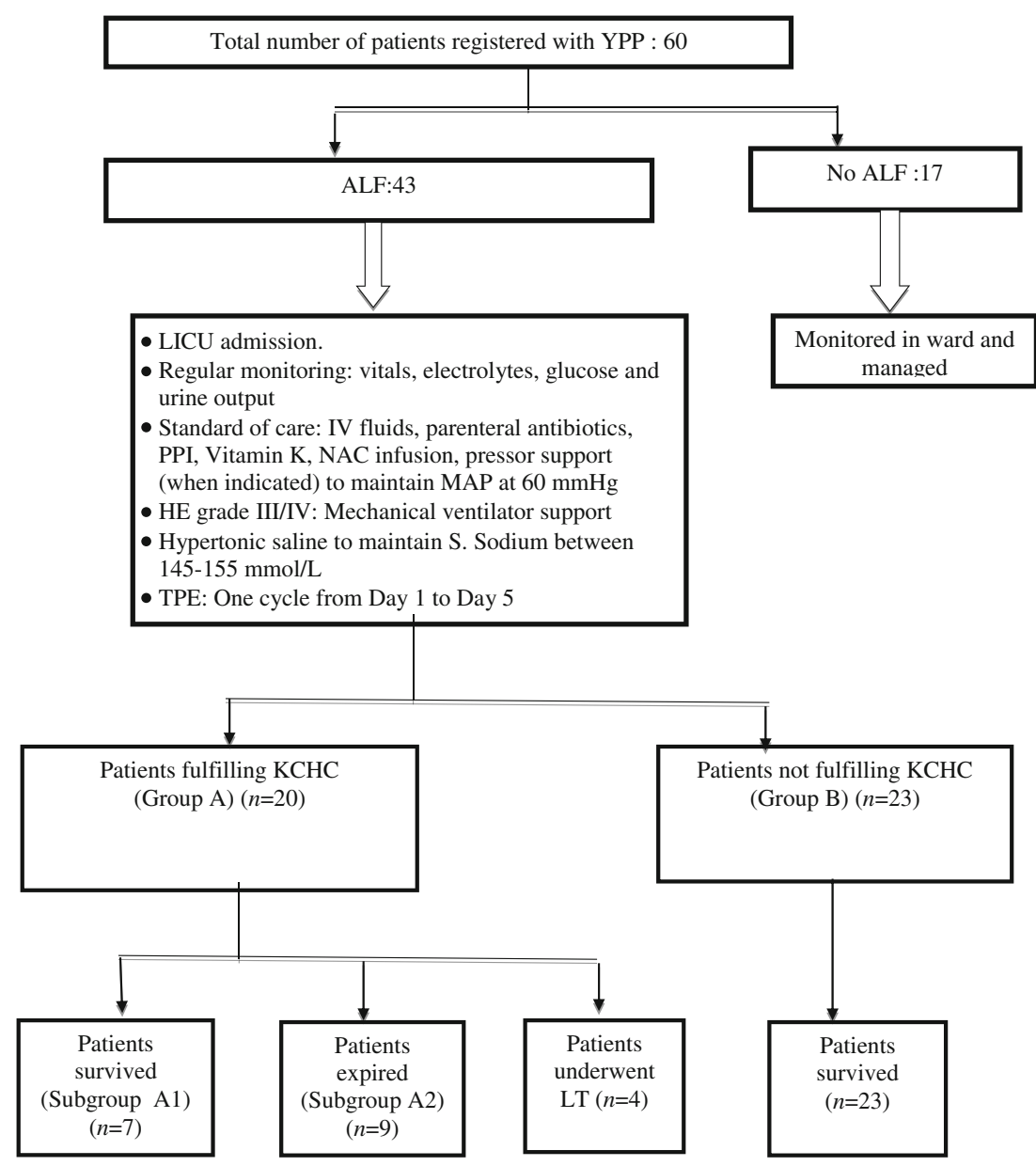

hematocrit. So, based on these variables, replacement volume varied between 2.5 to 4.0 litres. Pre-procedure serum calcium and potassium levels were checked, and intravenous correction was given to maintain normal serum potassium levels (3.5-4.5 $\mathrm{mmol} / \mathrm{L})$ and ionized calcium levels (1.0$1.3 \mathrm{mmol} / \mathrm{L}$ ).

King's College Hospital Criteria (KCHC) was used for listing patients for liver transplantation (LT). Those who fulfilled the criteria were categorized as Group A and the remaining as Group B.

For analysis, pre- and post-TPE details of liver biochemistry, prothrombin time/INR, arterial ammonia, and serum creatinine were collected. Total number of TPE cycles required and adverse events during TPE were recorded. Primary outcome was 3-month survival after TPE irrespective of LT.

Statistical analysis was performed using Statistical Package for Social Sciences (SPSS, Inc.; version 21.Chicago, IL, USA). Variables were classified into continuous and categorical. Since our data did not meet linearity, non-parametric tests like Wilcoxon signed-rank test and Mann-Whitney $U$ test were done to compare two related samples and independent samples, respectively. A $p$-value of $<0.05$ was considered statistically significant.

\section{Results}

Forty-three patients underwent TPE for YPP-induced ALF. Of them, 34 patients survived and 9 died. In survivor group, there were 20 males and 14 females. Mean age was $27.7 \pm$ 10.6 years. Mean number of TPE cycles per patient was 3.9 and mean replacement volume per cycle was $3.1 \pm 0.51$. In non-survivor group, there were 5 males and 4 females. Mean age was $26.9 \pm 9.5$ years. Mean number of TPE cycles per patient was 2.9 and mean replacement volume per cycle was $2.9 \pm 0.41$. There was statistically significant difference in alanine aminotransferase (ALT) $(p=0.042)$, ammonia $(p=$ $0.010)$, and mean number of TPE cycles $(p=0.039)$ between survivors and non-survivors (Table 1).

Out of 43 patients, further analysis was done based on $\mathrm{KCHC}$ for LT. Those who fulfilled KCHC for LT were classified in Group A and those who did not fulfill $\mathrm{KCHC}$ were classified in Group B. Twenty patients belonged to Group A and the remaining 23 belonged to Group B.

In Group A, there were 11 males and 9 females. Mean age was $29.7 \pm 11.4$ years. Mean number of TPE per patient was 3.2. TPE resulted in a statistically significant improvement in ALT ( $p$-value 0.000), aspartate aminotransferase (AST) 
Table 1 Comparison of baseline characteristics between survivors $(n=34)$ and non-survivors $(n=9)$

\begin{tabular}{lccc}
\hline Parameters & Survivors, mean $\pm \mathrm{SD}$ & Non-survivors, mean $\pm \mathrm{SD}$ & $p$-value \\
\hline Age $(\mathrm{y})$ & $27.7 \pm 10.6$ & $26.9 \pm 9.5$ & 0.821 \\
Number of TPE cycles & $3.9 \pm 1.5$ & $2.9 \pm 1.0$ & $0.039^{*}$ \\
Mean volume replaced per cycle $(\mathrm{L})$ & $3.1 \pm 0.5$ & $2.9 \pm 0.4$ & 0.207 \\
Pre-TPE total bilirubin $(\mathrm{mg} / \mathrm{dL})$ & $5.5 \pm 5.0$ & $6.3 \pm 3.2$ & 0.24 \\
Pre-TPE INR & $3.3 \pm 2.1$ & $4.3 \pm 3.0$ & 0.547 \\
Pre-TPE ALT $(\mathrm{U} / \mathrm{L})$ & $635 \pm 703$ & $1058 \pm 567$ & $0.042^{*}$ \\
Pre-TPE AST $(\mathrm{U} / \mathrm{L})$ & $1115 \pm 144$ & $1896 \pm 172$ & 0.159 \\
Pre TPE blood ammonia $(\mu \mathrm{mol} / \mathrm{L})$ & $61 \pm 39$ & $115 \pm 58$ & $0.010^{*}$ \\
\hline
\end{tabular}

Wilcoxon signed rank test, $* p<0.05$

TPE therapeutic plasma exchange, INR international normalized ratio, $A L T$ alanine aminotransferase, AST aspartate aminotransferase, $S D$ standard deviation $(p<0.001)$, and INR $(p=0.023)$ with no significant effect on levels of ammonia. In Group B, there were 14 males and 9 females. Mean age was $25.7 \pm 8.2$ years. Mean number of TPE per patient was 4.2. TPE showed statistically significant improvement in ALT $(p<0.018)$, AST $(p<0.012)$, INR $(p-$ value 0.000$)$, and blood ammonia levels $(p<0.034)$ (Table 2$)$. All 23 (100\%) patients survived.

Mean survival after completing TPE was $41.2 \pm 44.5$ days in Group A and 90 days in Group B. This difference was statistically significant $(U=80.5, p=0.001)$.

Subgroup analysis of Group A revealed 4 of 20 patients underwent successful LT. In the remaining 16 patients who did not receive LT, 7 survived without LT and 9 died while awaiting LT. Changes in biochemical parameters before and after TPE in both non-transplant survivors and non-transplant non-survivors in Group A are shown in Table 3. Among LTfree survivors, there was statistically significant improvement in biochemical parameters (INR, ammonia, and AST) after TPE as compared with non-survivors. Table 4 shows that, in non-survivors group, there were statistically significant high values of post-TPE INR $(p=0.012)$ and ammonia $(p=0.011)$ as compared with survivors.
There was no major procedure-related complication. Five patients developed hypotension and 2 minor allergic reactions; these were managed conservatively.

\section{Discussion}

In developed countries, YPP is common with industrial accidents, while in developing countries like India, this substance is often used as a suicidal agent and rarely by accidental ingestion. In the absence of an antidote for YPP, the definitive treatment for acute liver failure is liver transplantation (LT) following fulfillment of KCHC [10].

The clinical effects of YPP are classically divided in three stages. The initial gastrointestinal stage (within the first $24 \mathrm{~h}$ after ingestion) is characterized by vomiting, nausea, diarrhea, and abdominal pain. The laboratory tests at this stage are normal. Patients are usually symptom free in the second stage, which often lasts for 1 to 4 days or even more. Liver biochemistry shows gross derangement during this stage. The third stage (4-7 days) manifests as ALF with multiple organ dysfunction syndrome that is characterized by acute renal failure

Table 2 Biochemical changes after therapeutic plasma exchange in Group A (patients who fulfilled KCHC) and Group B (patients who did not fulfill $\mathrm{KCHC})$

\begin{tabular}{|c|c|c|c|c|c|c|}
\hline \multirow[t]{2}{*}{ Parameters } & \multicolumn{3}{|l|}{ Group A $(n=20)$} & \multicolumn{3}{|l|}{ Group B $(n=23)$} \\
\hline & Pre TPE, mean \pm SD & Post TPE, mean \pm SD & $p$-value & Pre TPE, mean \pm SD & Post TPE, mean \pm SD & $p$-value \\
\hline Total bilirubin (mg/dL) & $8.5 \pm 1.0$ & $9.0 \pm 0.5$ & 0.568 & $3.2 \pm 0.6$ & $3.9 \pm 0.7$ & 0.260 \\
\hline INR & $4.7 \pm 0.5$ & $2.9 \pm 0.4$ & $0.023 *$ & $2.5 \pm 0.3$ & $1.2 \pm 0.6$ & $0.001 * *$ \\
\hline ALT (U/L) & $1047 \pm 154$ & $301 \pm 62$ & $0.001 * *$ & $442 \pm 106$ & $172 \pm 37$ & $0.018^{*}$ \\
\hline AST (U/L) & $1959 \pm 395$ & $451 \pm 144$ & $0.001 * *$ & $227 \pm 79$ & $43 \pm 22$ & $0.012 *$ \\
\hline Blood ammonia $(\mu \mathrm{mol} / \mathrm{L})$ & $105 \pm 10$ & $92 \pm 13$ & 0.303 & $43 \pm 4$ & $33 \pm 3$ & $0.034 *$ \\
\hline
\end{tabular}

Wilcoxon signed rank test, ${ }^{*} p p<0.005,{ }^{*} p<0.05$. Pre-TPE values were measured before first cycle of TPE. Post-TPE values were measured on next day after last cycle of TPE

$T P E$ therapeutic plasma exchange, INR international normalized ratio, $A L T$ alanine aminotransferase, $A S T$ aspartate aminotransferase, $S D$ standard deviation, $K C H C$ King's College hospital criteria 
Table 3 Biochemical changes after therapeutic plasma exchange in survivors and non-survivors in Group A (patients who fulfilled KCHC)

\begin{tabular}{|c|c|c|c|c|c|c|}
\hline \multirow[t]{2}{*}{ Parameters } & \multicolumn{3}{|l|}{ Survivors $(n=7)$} & \multicolumn{3}{|l|}{ Non-survivors $(n=9)$} \\
\hline & Pre-TPE, mean \pm SE & Post TPE, mean \pm SE & $p$-value & Pre TPE, mean $\pm \mathrm{SE}$ & Post TPE, mean \pm SE & $p$-value \\
\hline Total bilirubin (mg/dL) & $9.8 \pm 2.2$ & $8.9 \pm 0.8$ & 0.686 & $6.3 \pm 1.1$ & $8.5 \pm 0.8$ & 0.072 \\
\hline INR & $5.3 \pm 2.2$ & $1.5 \pm 0.3$ & $0.004 * *$ & $4.3 \pm 1.0$ & $3.6 \pm 0.6$ & 0.602 \\
\hline ALT (U/L) & $1146 \pm 353$ & $112 \pm 20.45$ & $0.029 *$ & $1058 \pm 189$ & $517 \pm 107$ & $0.019 *$ \\
\hline AST (U/L) & $2212 \pm 799$ & $126 \pm 23.86$ & $0.042 *$ & $1896 \pm 590$ & $827 \pm 297$ & 0.077 \\
\hline Blood ammonia $(\mu \mathrm{mol} / \mathrm{L})$ & $81 \pm 16$ & $48 \pm 6$ & $0.045^{*}$ & $115 \pm 19$ & $136 \pm 24$ & 0.369 \\
\hline
\end{tabular}

Wilcoxon signed rank test, ${ }^{*} p<<0.005, * p<0.05$. Pre-TPE values were measured before first cycle of TPE. Post-TPE values were measured on next day after last cycle of TPE

$T P E$ therapeutic plasma exchange, INR international normalized ratio, $A L T$ alanine aminotransferase, $A S T$ aspartate aminotransferase, $S E$ standard error, KCHC King's College hospital criteria

with metabolic derangements, encephalopathy, coagulopathy, dysrhythmia, cardiogenic shock, and abnormal liver biochemistry. Central nervous system manifestation includes changes in mental status such as confusion, psychosis, hallucinations, and coma. LT is the only salvage during this stage [11].

High-volume plasma exchange (HVP) has been found to improve the transplant-free survival in ALF due to multiple etiologies in a multicentric study by Larsen et al. [5]. HVP may have a beneficial effect by delivery of physiologically important substances contained in fresh frozen plasma. The effect of HVP could also result from the removal of toxic factors such as circulating DAMPs (damage-associated molecular patterns). In our center, we perform low-volume plasma exchange as per the local experiences and observe that it performs especially well in toxin-mediated ALF such as YPP. This study was done with the intention to measure the safety and efficacy of TPE in terms of overall survival and transplant-free survival in ALF due to YPP. Due to limitations in the toxicological knowledge of the YPP, we could not measure serum toxin levels and the impact of TPE on the same.

Our study was similar to other studies which reported that YPP with ALF presentation was young (between 20 and 40 years) with a male gender predominance [9]. Previous study on 334 YPP in a tertiary care center in the same geographical location (Thanjavur, Tamilnadu, India) showed very high mortality rate $(76.2 \%)$ with standard medical care [12]. In contrast, our study reveals that the overall survival was $79 \%$ with TPE. We noticed that there was significant reduction in the values of ALT and ammonia following TPE among survivors than non-survivors. Moreover, interestingly nonsurvivors underwent less number of TPE when compared with survivors. This can be explained by our observation that nonsurvivors were hemodynamically very unstable to undergo further sessions of TPE.

Earlier study on YPP-induced ALF showed that there were no survivors without LT if they fulfill $\mathrm{KCHC}$ for LT [13]. In our study, among $\mathrm{KCHC}$ fulfilled 20 patients, 4 underwent liver transplant within $72 \mathrm{~h}$ of admission. In the remaining 16 listed for LT, 7 (43.8\%) patients improved and delisted from LT waiting list. In our study, $\mathrm{KCHC}$ had $100 \%$ sensitivity, $76.67 \%$ specificity, $65 \%$ positive predictive value (PPV), $100 \%$ negative predictive value (NPV), and $83.72 \%$ diagnostic accuracy (DA). This is the first study which shows LT-free survival in YPP-induced ALF patients by using TPE.

Among those who did not fulfill $\mathrm{KCHC}$, all (100\%) patients survived. In this group, TPE showed significant improvement in INR and ammonia level. Though the mean number of TPE cycles was more in this group, the difference between KCHC fulfilled (3.5) vs. non-fulfilled group (4.1) was not statistically significant $(p=0.096)$ after exempting LT patients.

Overall, TPE had no major adverse effects except hypotension $(11.62 \%)$ and mild allergic reactions $(4.65 \%)$, which were managed conservatively. As TPE is an extracorporeal device, it is not advisable if patients are hemodynamically
Table 4 Comparison of survivors and non-survivors in patients listed for liver transplantation (Group A) after therapeutic plasma exchange

\begin{tabular}{llll}
\hline Post TPE parameters & $\begin{array}{l}\text { Survivors }(n=7), \\
\text { mean } \pm \text { SD }\end{array}$ & $\begin{array}{l}\text { Non-survivors }(n=9), \\
\text { mean } \pm \text { SD }\end{array}$ & $p$-value \\
\hline Serum total bilirubin $(\mathrm{mg} / \mathrm{dL})$ & $8.9 \pm 2.0$ & $8.5 \pm 2.5$ & 0.66 \\
INR & $1.5 \pm 0.3$ & $3.6 \pm 1.8$ & $0.012^{*}$ \\
Blood ammonia $(\mu \mathrm{mol} / \mathrm{L})$ & $48 \pm 18$ & $136 \pm 71$ & $0.011^{*}$ \\
\hline
\end{tabular}

Wilcoxon signed rank test, $* p<0.05$

$T P E$ therapeutic plasma exchange, INR international normalized ratio, $S D$ standard deviation 
unstable and in presence of sepsis. Moreover, YPP will also cause acute severe pancreatitis and acute kidney injury along with ALF and those patients will not show benefit by either TPE or LT [14-16].

Based on our observations, despite the small sample size, we hypothesize that patients who had biochemical improvement after TPE may not eventually require emergency LT. TPE is an effective tool in our armamentarium in management of ALF following YPP and may even serve as bridge to liver transplant in those listed for LT. The present study is not without limitations. The small sample size and retrospective analysis may have resulted in a selection bias and skewed towards a favorable outcome with TPE. Multi-center, prospective study with a large sample size will provide definitive answers and throw light on the outcome of ALF patients following YPP.

We conclude that, in ALF due to YPP, TPE plays an important role and it is a safe, effective procedure in those who have not fulfilled KCHC for LT. Though our study reveals promising effect of TPE in KCHC fulfilled group, there is requirement of prospective, large volume, multi-center study to assess its efficacy.

\section{Compliance with ethical standards}

Conflict of interest JV, VJ, MKB, SM, RP, HN, VV, DS, PS, MJ, and $\mathrm{JV}$ declare that they have no conflict of interest.

Ethics statement The study was performed conforming to the Helsinki declaration of 1975, as revised in 2000 and 2008 concerning human and animal rights, and the authors followed the policy concerning informed consent as shown on Springer.com.

Disclaimer The authors are solely responsible for the data and the contents of the paper. In no way, the Honorary Editor-in-Chief, Editorial Board Members, the Indian Society of Gastroenterology or the printer/ publishers are responsible for the results/findings and content of this article.

\section{References}

1. Brent J, Wallace KL, Burkhart KK. Phosphorus. In: Brent J, Wallace KL, Burkhart KK, Phillips SD, Donovan JW, eds. Critical Care Toxicology - Diagnosis and Management of the Critically Poisoned Patient. Philadelphia: Elsevier Mosby; 2005. p. 851-61.
2. Tenenbein M. Position statement: whole bowel irrigation. American Academy of Clinical Toxicology; European Association of Poisons Centres and Clinical Toxicologists. J Toxicol Clin Toxicol. 1997;35:753-62.

3. Fernandez OU, Canizares LL. Acute hepatotoxicity from ingestion of yellow phosphorus-containing fireworks. J Clin Gastroenterol. 1995;21:139-42.

4. Liu CT, Chen TH, Cheng CY. Successful treatment of druginduced acute liver failure with high-volume plasma exchange. J Clin Apher. 2013;28:430-4.

5. Larsen FS, Schmidt LE, Bernsmeier C, et al. High-volume plasma exchange in patients with acute liver failure: An open randomised controlled trial. J Hepatol. 2016;64:69-78.

6. Lee WM, Stravitz RT, Larson AM. Introduction to the revised American Association for the Study of Liver Diseases Position Paper on Acute Liver Failure 2011. Hepatology. 2012;55:965-7.

7. European Association for the Study of the Liver. EASL Clinical Practical Guidelines on the management of acute (fulminant) liver failure. J Hepatol. 2017;66:1047-81.

8. Bhat S, Kenchetty KP. N-acetyl cysteine in the management of rodenticide consumption - life saving? J Clin Diagn Res. 2015;9: OC10-3.

9. Nalabothu M, Monigari N, Acharya R. Clinical profile and outcomes of rodenticide poisoning in tertiary care hospital. Int J Sci Res Publ. 2015;5:1-12.

10. Ates M, Dirican A, Ozgor D, et al. Living donor liver transplantation for acute liver failure in pediatric patients caused by the ingestion of fireworks containing yellow phosphorus. Liver Transpl. 2011;17:1286-91.

11. Talley RC, Linhart JW, Trevino AJ, Moore L, Beller BM. Acute elemental phosphorus poisoning in man: cardiovascular toxicity. Am Heart J. 1972;84:139-40.

12. Appavu V, Anthirias D, Dhanaraj H, et al. Yellow phosphorous poisoning: an observational study from a tertiary care hospital. J Evid Based Med Healthc. 2019;6:996-1002.

13. Saraf V, Pande S, Gopalakrishnan U, et al. Acute liver failure due to zinc phosphide containing rodenticide poisoning: clinical features and prognostic indicators of need for liver transplantation. Indian J Gastroenterol. 2015;34:325-9.

14. Kamarthi P, Subramani P, Gopu AV, Prasad R, Srinivasa C. Acute pancreatitis, hepatitis and bone erosion in acute yellow phosphorous compound poisoning - A rare complication. J Clin Diagn Res. 2016;10:DD03-5.

15. Soni JP, Ghormade PS, Akhade S, Chavali K, Sarma B. A fatal case of multi-organ failure in acute yellow phosphorus poisoning. Autops Case Rep. 2020;10:e2020146.

16. Türkmen Șamdanci E, Çakir E, Şahin N, Elmali C, Sayin S. Clinical and pathological findings on intoxication by yellow phosphorus after ingesting firework cracker: A rare Case of autopsy. Turk Patoloji Derg. 2016;32:51-3.

Publisher's note Springer Nature remains neutral with regard to jurisdictional claims in published maps and institutional affiliations. 